\title{
Subacute Sclerosing Panencephalitis in a Child with Human Immunodeficiency Virus Co-Infection
}

\author{
Pradeep Kumar Maurya, DM; Mayur Deepak Thakkar, MD; Dinkar Kulshreshtha, DM; \\ Ajai Kumar Singh, DM; Anup Kumar Thacker DM
}

\begin{abstract}
Subacute sclerosing panencephalitis is a fatal infectious disease of childhood caused by persistence of the measles virus in the brain. The effect of human immunodeficiency virus (HIV) co-infection on subacute sclerosing panencephalitis remains elusive and rare. We report a child who developed subacute sclerosing panencephalitis following a short latency period and a rapidly progressive course with HIV co-infection.
\end{abstract}

Keywords: Co-infection; HIV; Measles; Subacute sclerosing panencephalitis

$\mathrm{S}$ ubacute sclerosing panencephalitis (SSPE) is caused by a mutant measles virus and, in the majority of cases, eventually ends with a fatal outcome. It continues to be prevalent in developing countries despite widespread immunization programs. Human immunodeficiency virus (HIV) infection is another RNA virus that impairs the cellmediated immunity with consequent opportunistic infections. Co-infection with HIV in SSPE is a rare entity and the effect of each on the clinical course of the other may be perplexing. Two reports in the literature about this co-infection describe a fatal course in one and a favorable course in other. ${ }^{1,2}$ We report the clinico-radiological profile of a rare case of SSPE in a child with an HIV co-infection.

\section{Case Report}

A 10-year-old boy presented with a 4-month history of poor scholastic performance followed by sudden jerky movements of limbs that caused him to fall while walking. Gradually the frequency of the jerky movements increased, and he developed irritability. Additionally he developed progressive deterioration in sensorium leading to urinary incontinence. The patient had history of fever with generalized maculopapular rash 8 months prior, and he had recurrent ear discharge for one year. His immunization details were unavailable. His developmental milestones were normal. His mother died of prolonged febrile illness when he was 4-years-old; his father is living with an HIV-positive status. On neurological examination, the patient was confused. His pupils were equally reactive to light and fundus was normal. Motor examination revealed spasticity and brisk deep tendon jerks with extensor plantars. Asymmetric myoclonic jerks were present, involving the trunk and limbs (left greater than right) recurring at 4 to 6 seconds interval. $\mathrm{He}$ also had associated orofacial dyskinesia. The remainder of the systemic examination was unremarkable. In view of the progressive cognitive decline and myoclonic jerks, the possibility of SSPE was considered and investigated accordingly. Electroencephalography (EEG) revealed generalized periodic sharp and slow wave complexes with 1:1 relation with myoclonus with background slowing (figure1). His cerebrospinal fluid (CSF) analysis showed 10 cells $/ \mu \mathrm{L}$; all were lymphocytes, mildly elevated proteins $61 \mathrm{mg} / \mathrm{dL}$ (normal $<45 \mathrm{mg} / \mathrm{dL}$ ), with normal CSF sugar $(58 \mathrm{mg} / \mathrm{dL})$. His serum and CSF were positive for IgG anti-measles antibody by enzyme-linked immunosorbent assay (ELISA), while $\operatorname{IgM}$ was negative, suggestive of chronic measles infection. However, quantitative estimation of IgG in serum and CSF could not be obtained. Polymerase chain reaction for John Cunningham virus in the CSF was negative, and the CSF was also negative for bacteria, tuberculosis, herpes, varicella zoster, and cryptococcal infection. His serum for anti-HIV 1 antibody was positive. The hepatitis $\mathrm{B}$ and $\mathrm{C}$ antibody titers were negative. His CD4 count was $900 / \mu \mathrm{L}$ (normal; 410$1590 / \mu \mathrm{L})$. Magnetic resonance imaging (MRI) of the brain on axial T2 weighted and fluid attenuation inversion recovery images showed hyper-intense subcortical lesions in bilateral frontal and parietal regions without restriction on diffusion weighted imaging and no contrast enhancement suggestive of demyelination (figure 2). The patient was treated with clonazepam, sodium valproate, and isoprinosine. He was registered at the antiretroviral therapy (ART) center and
Corresponding Author: Pradeep Kumar Maurya, DM, Department of Neurology, Dr. Ram Manohar Lohia Institute of Medical Sciences, Lucknow 226010 INDIA, Email: pkm730@gmail.com
Received: May 18, 2016

Revised: September 17, 2016

Accepted: September 26, 2016

doi: $10.3121 / \mathrm{cmr} .2016 .133 \mid$ 


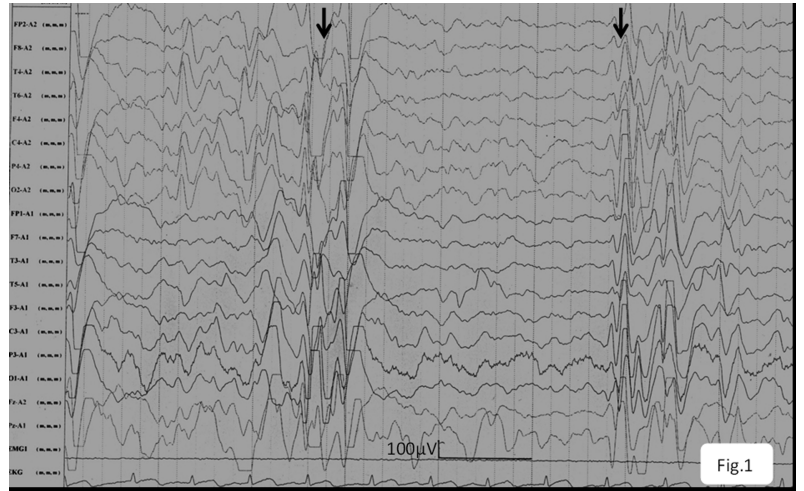

Figure 1. Electroencephalography of the patient (monopolar montage) shows bi- and triphasic high amplitude (200$600 \mu$ volt) sharp and slow wave complexes (arrowheads) of 1 second duration recurring at 4 to 6 seconds interval in a symmetric and synchronous fashion with slow background. These discharges are in 1:1 relation with myoclonic jerks.

treated with antiretroviral drugs. He displayed mild improvement in sensorium with reduced myoclonic jerks at the 6-month follow-up. There was a reduction in CD4 count $(470 / \mu \mathrm{L})$ noted at 6 months, although it was within normal range.

\section{Discussion}

Apart from SSPE, measles can be complicated by acute measles encephalitis, acute disseminated encephalomyelitis, and inclusion body encephalitis; of these, SSPE is the most serious. The effect of HIV on development of SSPE has not been reported often. Perinatally-acquired HIV or a child born to an HIV positive mother may have a short incubation period for developing SSPE, as it has been reported in two children. One HIV positive child developed SSPE at 21 month and one HIV negative child whose mother was HIV positive developed SSPE at 4 years old. ${ }^{3}$ Our patient had both of the above features; perinatally acquired HIV and SSPE developed at a short incubation period.

SSPE may have a fulminant course in patients with HIV, as reported in a 17 year old male who perinatally acquired the infection, and who died within 12 weeks of onset of SSPE. ${ }^{1}$ Another child with HIV developed measles at age 5 months and later developed SSPE at 14 years - a long latency period-but is reported to remain stable on ART at 7 months of follow up. ${ }^{2}$ SSPE has also been reported in a 7-year-old child with HIV who was stable on ART for 2 years. ${ }^{4}$ Usually the latency between measles infection and disease onset is 6 to 8 years followed by progression over 1 to 3 years, leading to death. ${ }^{5}$ However the variability in the onset, latency, and clinical progression has been reported to be influenced by various factors related to host disease interaction. ${ }^{5}$ Both SSPE and HIV escape the immune surveillance for a variable time before the disease manifests. Genetic polymorphism of individuals may lead to altered cellular response to a common antigen in SSPE. ${ }^{6}$
The short latency in our patient was likely due to HIV-related impaired cell-mediated immunity. Our patient also had rapid progression of symptoms leading to marked cognitive decline with bladder and bowel dysfunction within 4 months of symptoms onset. The EEG was typical in our case, showing classical periodic complexes at 4 to 6 second intervals as described by Cobb. ${ }^{7}$ Our case fulfilled three of five criteria for the diagnosis of SSPE. ${ }^{8}$ The lesion on the brain MRI in our case can occur in HIV encephalitis or progressive multifocal leukoencephalopathy. The polymerase chain reaction for John Cunningham virus in the CSF was negative in our patient excluding the possibility of progressive multifocal leukoencephalopathy. In HIV encephalitis, focal hyperintense lesions develop in the frontal lobe, which progress and become generalized without enhancement and mass effect. ${ }^{9}$ Our patient was classified at World Health Organization clinical stage II, and ART was initiated. There was a decline in CD4 count observed at the 6-month follow up, despite mild improvement in symptoms. However, the long term effect of ART remains to be observed. Our case highlights that HIV co-infection, even with a normal CD4 count, can lead to shorter latency and rapid progression of SSPE. The literature, however, is scarce with respect to this serious co-infection.

In summary, early HIV infection with normal CD4 count can alter the clinical behavior of SSPE, possibly due to impaired cell-mediated immunity. A patient presenting with atypical features of SSPE should make one vigilant for associated co-infection with HIV.

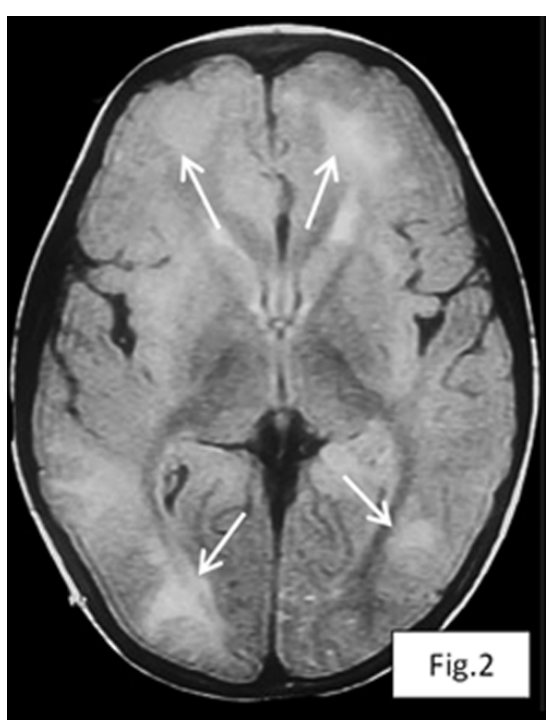

Figure 2. Magnetic resonance imaging of brain (axial images) shows subcortical white matter hyperintense signal in bilateral frontal and parieto-occipital region on fluid attenuation inversion recovery (FLAIR) sequence (arrowheads). 


\section{References}

1. Sivadasan A, Alexander M, Patil AK, Balagopal K, Azad ZR.

Fulminant subacute sclerosing panencephalitis in an individual with a perinatally acquired human immunodeficiency virus infection. Arch Neurol. 2012;69(12):1644-1647.

2. Muthusamy K, Yoganathan S, Thomas MM, Alexander M, Verghese VP. Subacute sclerosing panencephalitis in a child with human immunodeficiency virus (HIV) infection on antiretroviral therapy. Ann Indian Acad Neurol. 2015;18(1):96-98.

3. Koppel BS, Poon TP, Khandji A, Pavlakis SG, Pedley TA. Subacute sclerosing panencephalitis and acquired immunodeficiency syndrome: role of electroencephalography and magnetic resonance imaging. J Neuroimaging. 1996;6(2):122-125.

4. Gowda VKN, Sukanya V, Shivananda. Acquired immunodeficiency syndrome with subacute sclerosing panencephalitis. Pediatr Neurol. 2012;47(5):379-381.

5. Garg RK. Subacute sclerosing panencephalitis. Postgrad Med J. 2002;78(916):63-70.

6. Yentür SP, Gürses C, Demirbilek V, et al. Alterations in cellmediated immune response in subacute sclerosing panencephalitis. J Neuroimmunol. 2005;170(1-2):179-185.

7. Cobb W. The periodic events of subacute sclerosing leucoencephalitis. Electroencephalogr Clin Neurophysiol. 1966;21(3):278-294.

8. Dyken PR. Subacute sclerosing panencephalitis. Current status. Neurol Clin. 1985;3(1):179-196.

9. Thurnher MM, Thurnher SA, Schindler E. CNS involvement in AIDS: spectrum of CT and MR findings. Eur Radiol. 1997;7(7):1091-1097. 